\title{
Pro-inflammatory cytokine; tumor-necrosis factor-alpha (TNF- $\alpha$ ) inhibits astrocytic support of neuronal survival and neurites outgrowth
}

\author{
Ebtesam M. Abd-El-Basset
}

Department of Anatomy, Faculty of Medicine, Kuwait University, Kuwait City, Kuwait Email: ebtesam@hsc.edu.kw

Received 16 June 2013; revised 16 July 2013; accepted 31 July 2013

Copyright (C) 2013 Ebtesam M. Abd-El-Basset. This is an open access article distributed under the Creative Commons Attribution License, which permits unrestricted use, distribution, and reproduction in any medium, provided the original work is properly cited.

\section{ABSTRACT}

Reactive astrogliosis has been implicated in the failure of axonal regeneration in adult mammalian Central Nervous System (CNS). It is our hypothesis that inflammatory cytokines act upon astrocytes to alter their biochemical and physical properties, which may in turn be responsible for the failure of neuronal regeneration. We have therefore examined the effect of tumor-necrosis factor-alpha (TNF- $\alpha$ ) on the ability of astrocytes to support the survival of the cortical neurons and the growth of the neurites. Mouse astrocytes and cortical neuronal cultures were prepared. It was observed that when neurons were cultured in absence of astrocytes only a few of them grew and survived only for 5 - 6 days. These neurons had small cell bodies and few, short neurites. However, when the same numbers of neurons were cultured on the top of astrocytes, more neurons grew and survived up to 16 18 days. They had bigger cell bodies and many long branched neurites that formed anestamosing networks. The neurons then coalesced and the neurites formed thick bundles. When the same numbers of neurons were grown on the top of astrocytes pretreated with TNF- $\alpha$, few neurons survived up to 13 days. The neurites of the survived neurons were shorter than neurites of neurons grown on normal astrocytes and did not form bundles. In addition, TNF-a stimulated the expression of glial fibrillary acidic protein (GFAP) by astrocytes. These results support that the pro-inflammatory cytokine, TNF- $\alpha$ modulates the gliosis and that the astrocytic cell supports neuronal survival and neurite outgrowth.

Keywords: Astrocytes; Neurons; Cytokines; Tumor-Necrosis Factor-Alpha

\section{INTRODUCTION}

Astrocytes support the CNS neurons, by controlling the microenvironment surrounding the neurons, picking up any excess neurotransmitters or ions that are secreted from the neurons during conduction of nerve impulses and metabolic activity $[1,2]$. Astrocytes are also important in secreting several trophic factors, vital to the survival of neurons $[3,4]$. Astrocytes can promote neurite outgrowth both in vitro and in vivo. They express receptors for a variety of growth factors and neurotansmittors [5]. Nerve growth factors production in astrocytes is regulated by various cytokines including TNF- $\alpha$ [6]. In addition, astrocytes play a crucial part in the antioxidant defence of the brain [7].

However, in cases of insult to the CNS, astrocytes begin to accumulate at the margin of the injury forming a layer that interfaces between the injury and intact tissues, which become known as the glial scar or reactive astrogliosis [8]. Reactive astrogliosis is characterized by an increase in the amount of GFAP which is regulated by hormones, cytokines, and growth factors [9,10]. Although the functional role of the glial scar is not yet completely understood, it has been suggested that the scar inhibits nerve regeneration by acting as a physical barrier that neurons cannot penetrate $[8,11]$. However, the formation of the glial scar can also be beneficial in isolating the damaged region and in preventing the spread of the inflammatory response to intact neuronal tissue $[12,13]$ thereby helping to limit tissue degeneration and preserving function after CNS injury. In addition, recent data showed axon regeneration through scars and into sites of chronic spinal cord injury [14]. All these data emphasize that reactive astrocytes play a role in wound healing and restoring CNS function at the site of injury. The data also indicate the importance of preserving astrocyte populations $[13,15,16]$. 
During injury to the CNS, astrocytes are also responsible for the secretion of cytokines, such as interleukin-1 (IL-1), interleukin-6 (IL-6) and TNF- $\alpha$ [17]. Cytokines are responsible for certain changes that take place in the nervous system [18]. It is known that the immune system and the nervous system communicate with each other through the sharing of biochemical informational substances and their receptors, which is referred to as neural-immune interactions. For instance, several neurotransmitters, neuroendocrine hormones and even nerve growth factors are produced by immune cells and clearly play an important role in the nervous system $[4,6]$. The CNS has its own resident immune system in which glia cells (astrocytes and microglia) not only serve supportive and nutritive roles for neurons but also engage from time to time in several inflammatory processes that defend the CNS from pathogens and help it to recover from stress and injury. These normal glia functions are kept by normal activity of neurons [19]. The immune status of the CNS is strictly regulated in healthy brain in which the immune response is kept to a minimum by activity of neurons. Loss of neurons or their physiological activity would render the CNS recognizable by invading immune cells and can sometimes result in a more severe neuroinflammatory cycle that promotes neurodegenerative diseases [20].

TNF- $\alpha$, a potent proinflammatory molecule, is mainly produced by astrocytes in the CNS. During brain injury or inflammatory processes TNF- $\alpha$ is produced by activated microglia, astrocytes and macrophages [17,21]. In culture, astrocytes secret TNF at low level but the secretion is increased after treatment with inflammatory stimuli such as interferon-gamma combined with IL-1beta. TNF- $\alpha$ promotes the proliferation of astroglia and microglia and therefore may be involved in pathological processes such as astrogliosis and demyelination [22]. TNF- $\alpha$ triggers downstream signaling cascades that control a number of cellular processes related to cell viability, gene expression, ion homostasis and synaptic integrity [23]. It should be indicated that there is limited information available on the effect of TNF- $\alpha$ on astrocytes-cortical neurons interactions. It is our hypothesis that inflammatory cytokines act upon astrocytes to alter their biochemical and physical properties, which may in turn be responsible for the failure of neuronal regeneration. We have therefore examined the effect of TNF- $\alpha$ on the ability of astrocytes to support the survival of the neurons and the growth of the neurites in mixed neuronastrocyte cultures. The latter provides a valuable in vitro model system for studying the direct interactions between neurons and astrocytes.

\section{MATERIALS AND METHODS}

Animal care followed the recommendations of NIH
Guidelines for Care and use of laboratory Animals and the Guide for the Care and Use of Laboratory animals (Kuwait University-Faculty of Health Publication). All efforts were made to minimize animal suffering, to reduce the number of animals used, and to utilize alternatives to in vivo techniques.

\subsection{Astrocyte Cultures}

The astrocyte cultures were prepared according to the method of [24]. The cerebral hemispheres of newborn $\mathrm{Balb} / \mathrm{c}$ mice were isolated aseptically and the meninges were removed. The neopallia was dissected out and then forced through a sterile $75 \mu \mathrm{m}$ Nitex mesh. The cells were suspended in a modified Eagle's Minimum Essential Medium (mMEM) containing 5\% horse serum. For culturing, $5 \times 104$ nigrosine-excluding cells were plated in $60 \mathrm{~mm}$ Petri dishes containing $22 \times 22 \mathrm{~mm}$ glass coverslips in a total volume of $3 \mathrm{ml}$ growth medium. Aliquots of $5 \times 106$ nigrosine-excluding cells were plated in $75 \mathrm{~cm}^{2}$ culture flasks. The cultures were incubated at $37^{\circ} \mathrm{C}$ in a humidified atmosphere of $5 \% \mathrm{CO}_{2}$ in air. After 3 days of incubation the growth medium was removed, the cell debris and non-attached cells were washed off and fresh medium was added and was changed every 2 3 days. After 10 days some culture plates were incubated for 5 days and culture flasks for 2 and 8 days in medium to which 200 international units $(\mathrm{U}) / \mathrm{ml}$ of TNF- $\alpha$ (Sigma, St. Louis, MO) had been added. The new medium was also changed every $2-3$ days.

\subsection{Neuronal Cultures}

The cerebral neuronal cultures were prepared according to the method of [25]. The outer parts of the brain of embryonic mice foetuses (E 15) were used. The cells of the brains were separated in $0.25 \%$ trypsin in Puck's solution for $5 \mathrm{~min}$. at room temperature. Horse serum was added to stop the trypsin action, and the tissue was triturated by pipetting up and down. The suspensions were centrifuged at $1000 \mathrm{rpm}$ for $5 \mathrm{~min}$. After removal of supernatant, the cells were resuspended in mMEM containing 30 mili-moles $(\mathrm{mM})$ glucose and filtered through a sterile $75 \mu \mathrm{m}$ Nitex mesh. For culturing $5 \times 104$ nigrosine-excluding cells were plated in $60 \mathrm{~mm}$ Petri dishes containing $22 \times 22 \mathrm{~mm}$ glass coverslips coated with poly-L-lysine (Sigma) in a total volume of $3 \mathrm{ml}$ growth medium. After incubation in serum-free medium for 15 min. at $37^{\circ} \mathrm{C}$, the culture was rinsed once with fresh medium and incubated in freshly prepared medium containing $5 \%$ hourse serum and 30 mili-moles $(\mathrm{mM})$ glucose, with changes of medium every 2 - 3 days.

\subsection{Neuron-Astrocyte Mixed Cultures}

The astrocyte cultures were maintained for 10 days in 
medium containing 5\% serum. The neuronal cells were suspended and prepared as described above and the same numbers of neurons $5 \times 104$ were plated onto the astrocyte culture. After $15 \mathrm{~min}$ of incubation at $37^{\circ} \mathrm{C}$, the culture was rinsed once and then fed with fresh medium containing 5\% horse serum and $30 \mathrm{mM}$ glucose. Some of the 10 days old astrocyte culture were treated for 5 days with $200 \mathrm{U} / \mathrm{ml}$ of mouse TNF- $\alpha$ then washed. The same numbers of neurons $5 \times 104$ were placed into the TNF- $\alpha$ pre-treated astrocyte culture and incubated for another 20 days.

\subsection{Immunofluorescence}

The neurons grow on the top of astrocytes and on the top of TNF- $\alpha$ pre-treated astrocytes were fixed for $4 \mathrm{~min}$ with $-20^{\circ} \mathrm{C}$ methanol. The fixed cells were treated first with primary mouse monoclonal antibody to neurofilament (NF-68) (Sigma, St. Louis, MO) diluted 1:200 in PBS, for $45 \mathrm{~min}$. The cells were then treated with the secondary antibody; fluorescein-conjugated donkey antimouse IgG (Jackson Immuno reseach Lab. Inc. Baltimore Pike) diluted 1:100 in PBS for $45 \mathrm{~min}$. For controls the primary antibody was omitted. All the procedures were carried out at room temperature. After application of each antibody the preparations were washed 3 times for $5 \mathrm{~min}$. each in PBS. The cells were mounted in 50\% glycerol in PBS, pH 7.8 and examined in Olympus photomicroscope equipped with mercury vapor lamp, epifluorescence optics and appropriate interference filters.

\subsection{Morphological Studies and Neurite Outgrowth Essay}

The morphological changes in neurons after 2, 5, 7, 13, 17,20 days of cultures will be examined by phase contrast microscopy.

To assess the numbers of survived neurons, the numbers of neurons extending neurites longer than the two cell body diameter were counted in 8 randomly chosen microscopic fields using a $\times 20$ objective lens of Ziss phase contast microscope. The fields were examined from the center of each coverslip inside the tissue culture dish. 3 dishes were examined from each experiment. 3 separate independent experiments were used. The length of the neurites from the above neurons was measured using imaging analysis program (Image J, version 1.04b, Wayne Rasband, NIH). In each experiment 50 - 60 neurites were measured.

\subsection{Polyacrylamide Gel Electrophoresis (PAGE) and Immunobloting}

Flasks of TNF- $\alpha$ treated astrocytes for 2 and 8 days and control of non treated astrocytes were washed three times in PBS, scraped off with a rubber policeman and collec- ted in PBS. The cells were immediately sonicated and the protein concentration was determined according to the method of [26]. The sonicated cells were dissolved in $10 \%$ SDS-containing sample buffer and boiled for $3 \mathrm{~min}$. The same amount of protein $(20 \mu \mathrm{g} /$ well $)$ from each sample was analyzed electrophoretically in 10\% SDSPAGE gel according to the method of [27]. The proteins in the gel were transferred to nitrocellulose sheets by a modification of the method of [28]. After transfer, the nitrocellulose sheets were incubated for $1 \mathrm{~h}$ with $3 \%$ bovine serum albumin (BSA) in PBS. The nitrocellulose sheets were then incubated overnight at room temperature with rabbit polyclonal antibody to GFAP, diluted 1:500 in PBS containing 3\% BSA, then followed by affinity-purified goat anti-rabbit IgG conjugated to horseradish peroxidase. The color was developed using freshly prepared $0.05 \%$ 4-chlor-1-naphthol and $0.015 \% \mathrm{H}_{2} \mathrm{O}_{2}$ in PBS. The reaction was stopped by washing in tap water. All the chemical and materials for electrophoresis and immunobloting were purchased from BioRad (Missisauga, Ont, Canada). Immunoblots of five independent experiments were scanned using the snapescan 1212 scanner and Adobe Photoshop 5.0 program. The relative levels of GFAP expression were determined by analyzing the pixel intensity of the bands using an imaging analysis program (Image J, version 1.04b, Wayne Rasband, NIH). The percentage of the protein expression was calculated in the following manner. The average background protein levels in the lane, excluding the bands, were first subtracted from both the control and treatment bands. The $\%$ of the decrease or increase in intensity (I) of TNF- $\alpha$ treated cells (T) compare to the control (C) was calculated as follows:

- $\%$ of decrease in the intensity $=100 \times($ Mean I of C Mean I of T)/Mean I of C;

- $\%$ of increase in the intensity $=100 \times($ Mean I of T Mean I of C)/Mean I of C.

\subsection{Statistical Analysis}

Experiments were performed at least three times on different cell preparations. The data about the number of the survived neurons and the length of the neuritis of neuron cultured alone, on top of astrocytes and on top of TNF- $\alpha$ pre-treated astrocytes were analyzed with oneway Analysis of Variance (ANOVA) which rejected the null. P-values $<0.05$ were considered statistically significant. A post-hoc bonferroni's test was used to compare the three groups individually.

\section{RESULTS}

\subsection{The Effect of Asrtocytes on the Survival of Neurons}

When the neurons were cultured alone without astrocytes, 
few of them grew and survived only for 5 - 6 days (Figure 1a). These neurons had small cell bodies and a few short neurites. However, when the same numbers of neurons were cultured on the top of astrocytes, more neurons grew (Figure 1b) and survived up to $16-18$ days. They had bigger cell bodies and many long branched neurites that formed anastomosing networks (Figure 2a). The neurons then coalesced and the neurites formed thick bundles (Figure 3a).

\subsection{The Effect of TNF- $\alpha$ on the Survival of Neurons}

When neurons were culture on the top of astrocytes which were pre-treated with TNF- $\alpha$ for 5 days, few neu-
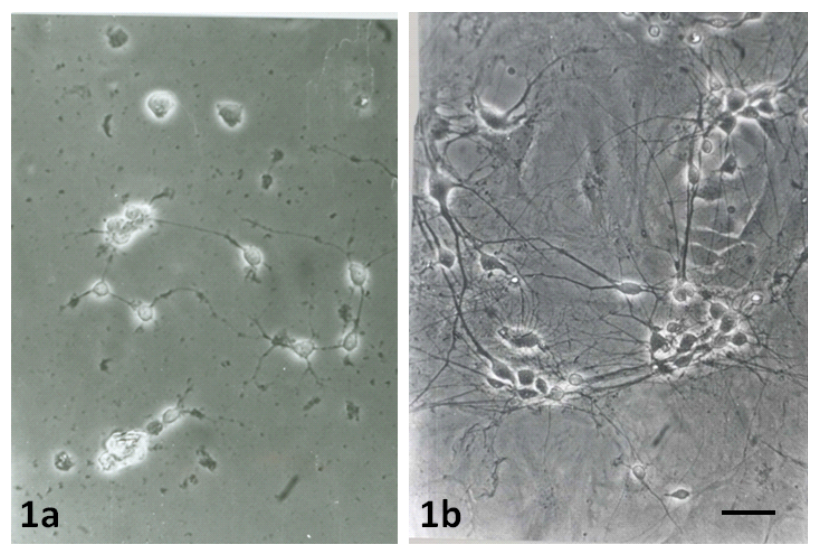

Figure 1. Phase contrast image of 5 days old neuronal cultures growing without astrocytes (1a). Image shows few numbers of neurons with small cell bodies and short neurites. Phase contrast image of 5 days old neuronal cultures on top of normal astrocytes (1b) showing many numbers of neurons with long neurites. Scale bar is $50 \mu \mathrm{m}$.
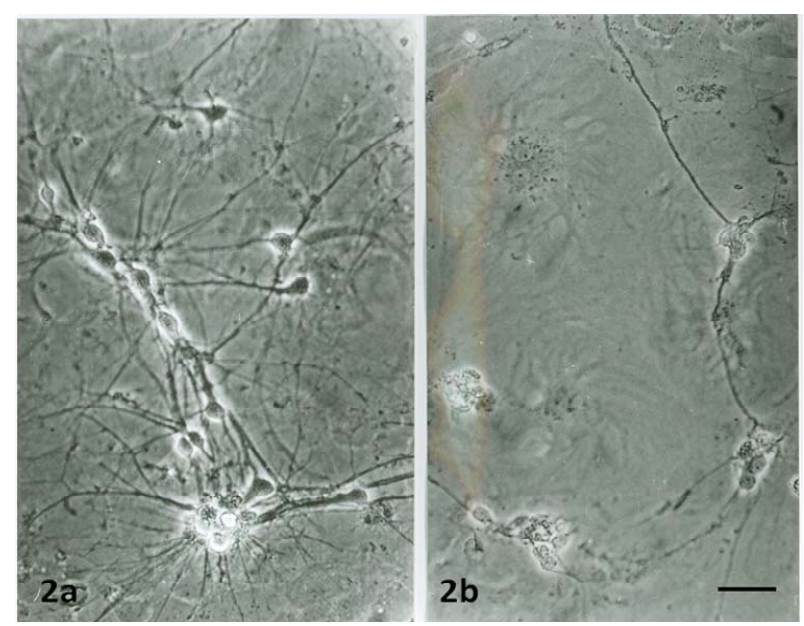

Figure 2. Phase contrast images of 7 days old neuronal cultures on the top of normal astrocytes (2a) showing many neurons grow and extend long neurites. Neurons on top of TNF- $\alpha$ pre-treated astrocytes (2b) showing less survived neurons than when they grow on non-treated astrocytes. Scale bar is $50 \mu \mathrm{m}$. rons survived in 7 days culture (Figure 2b) and only up to 13 days (Figure $\mathbf{3 b}$ ). The neurites did not form bundles (Figure 3b) as compare to that of neurons grew on top of nontreated astrocyes (Figure 3a).

\subsection{Immunofluorescence Staining}

When neurons and their neurites were stained for neurofilaments, many neurons and neurites were stained in 7 days old neuronal cultures grew on the top of normal astrocytes (Figure 4a) compare to the neurons grew on top of pre-treated astrocytes with TNF- $\alpha$ (Figure 4b).
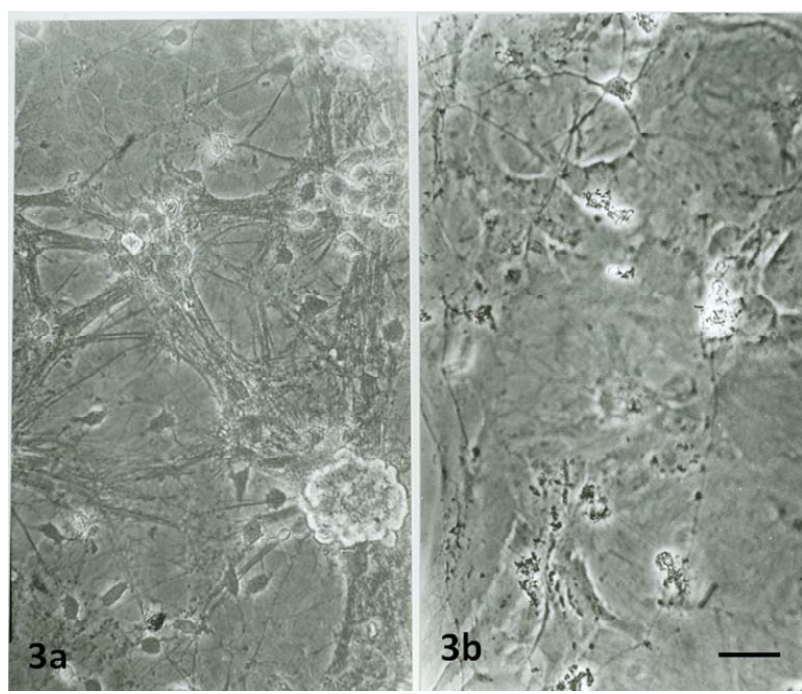

Figure 3. Phase contrast images of 13 days neuronal cultures on top of normal astrocytes (3a) showing many neurons form group together and the neurites form bundles. Neurones on the top of TNF- $\alpha$ pre-treated astrocytes (3b) showing few neurons survived. Scale bar is $50 \mu \mathrm{m}$.
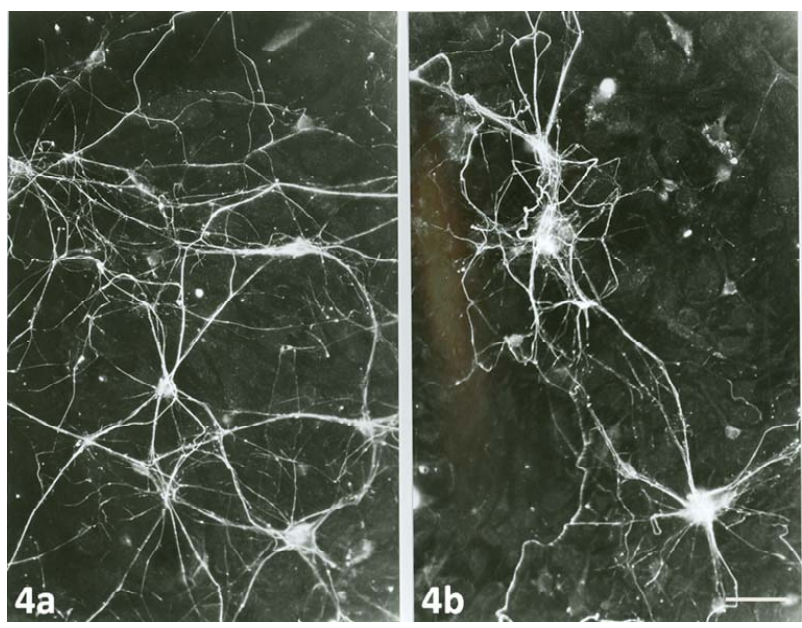

Figure 4. Immunofluorescence staining with monoclonal antibody to neurofilaments (68) of 7 days old neuronal cultures on the top of normal astrocytes (4a) and on pre-treated astrocytes with TNF- $\alpha$ (4b) showing that many neurons with long neurites in $4 \mathrm{a}$ compare to $4 \mathrm{~b}$. Scale bar is $20 \mu \mathrm{m}$. 


\subsection{The Neuronal Survival Essay}

In 5 days old culture, when neurons were cultured alone without astrocytes only few of them grew $(11 \pm 2)$. When the same numbers of neurons were cultured on the top of astrocytes $73 \pm 4$ neurons survived. However, when the same numbers of neurons were cultured on the top of TNF- $\alpha$ pre-treated astrocytes the number of survived neurons decreased to about $40 \pm 3$ neurons. In the older cultures $(15,17$ days old) no neurons survived when cultured alone as well as when cultured on top of TNF- $\alpha$ pre-treated astrocytes. However, when they were cultured on top of normal astrocytes about $35 \pm 5$ neurons survived in the 13 days old culture, $32 \pm 2$ neurons survived in the 15 days old culture, and $23 \pm 2$ neurons in the 17 days old culture (Figure 5). The data showing the number of the survived neurons cultured alone, on top of astrocytes and on top of TNF- $\alpha$ pre-treated astrocytes were analyzed with one-way Analysis of Variance (ANOVA) which rejected the null and showed that there was a significant difference between the three groups (Pvalues $<0.001)$. A post-hoc bonferroni's test was used to compare the three groups individually and showed that there was a significant difference between each of the three groups with a P-value of $<0.001$ for all groups.

\subsection{The Neurites Outgrowth Essay}

Measurement of the length of the neurites using the Image $\mathrm{J}$ analysis program showed that neurons cultured on the top of normal astrocytes have the longest neurites with progressive increases in the length with the days in culture compared to the neurons cultured alone (without astrcoytes) or neurons cultured on top of TNF- $\alpha$ pretreated astocytes. It should be notes that the sudden drop

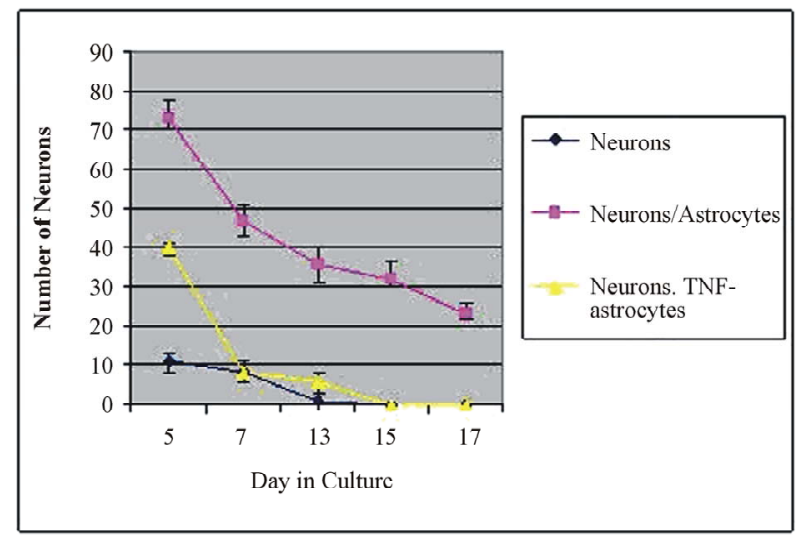

Figure 5. Graph showing number of neurons in three different conditions: neuron culture alone, neurons on the top of astrocyte and neurons on the top of TNF- $\alpha$ pre-treated astrocytes. Note the significant increase in the number of neurons growing on the top of astrocytes (P-values $<0.001)$ and longer period of survival (17 days) compared to the neuron growing alone or on the top of TNF- $\alpha$ pre-treated astrocytes. (to zero) in the length of the neurites in both neurons cultured alone (at day 7) or neurons cultured on top of TNF- $\alpha$ pre-treated astrocytes (at day 13) was due to the absence of the neurons and therefore the absence of neurites (Figure 6). The data about the length of the neurites of neurons cultured alone, on top of astrocytes and on top of TNF- $\alpha$ pre-treated astrocytes were analyzed with one-way Analysis of Variance (ANOVA) which rejected the null and showed that there was a significant difference between the three groups (P-values $<0.001)$. A post-hoc bonferroni's test was used to compare the three groups individually and showed that there was a significant difference between each of the three groups with a $\mathrm{P}$-value of $<0.001$ for all groups.

\subsection{Immunobloting}

To determine the effect of TNF- $\alpha$ on the expression of GFAP in astrocytes, proteins from 2 and 8 days TNF- $\alpha$ treated astrocytes and control astrocytes (non-treated) were separated by SDS-PAGE, transferred to nitrocellulose sheets and treated with antibody to GFAP (Figure 7). Quantification of the western blots of five independent experiments showed that the amount of GFAP in 2 -day treated astrocytes increased to about $125 \%$ of control, whereas, the amount of GFAP in 8-day treated astrocytes increased to $135 \%$ of control.

\section{DISCUSSION}

This study shows that neuronal survival and growth depended on astrocytes. Similarly, the fact that TNF- $\alpha$ is shown to play an inhibitory role on neuronal survival and growth shows that cytokines play important modulatory roles in the neuronal/astrocytic relationship. It is clear

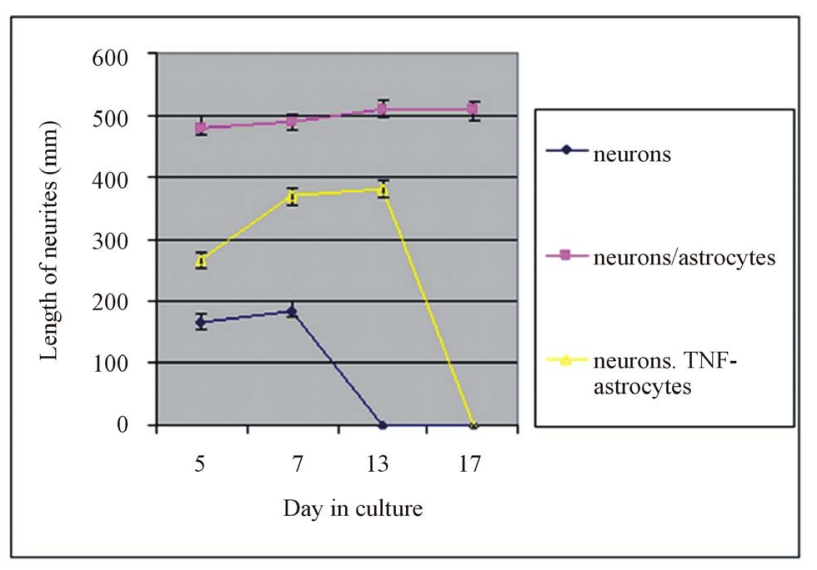

Figure 6. Graph showing the lengths of the neutites in 3 different conditions: neurons cultured alone, neurons growing on the top of astrocytes, and neurons growing on the top of TNF- $\alpha$ pre-treated astrocytes. Note the significant increase lengths of the neutites of the neurons growing on top of astrocytes (Pvalues $<0.001)$. These lengths were magnified by $1220 \times$. 


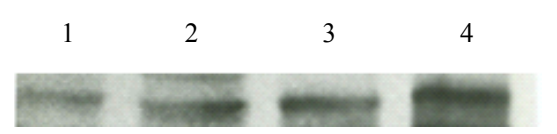

Figure 7. Immunobloting of the transferred total protein extract of control non-treated astrocytes (lane 1); 2 days TNF- $\alpha$ treated astrocytes (lane 2); 8 days control non-treated astrocytes (lane 3) and 8 days TNF- $\alpha$ treated cells (lane 4) stained with antibodies to GFAP.

that both astrocytes and cytokines play very important role in determining the milieu of the neurons, which is vital in the correct development, proliferation and reaction to CNS insult.

Astrocytes support the growth of neurons, when neurons were cultured on top of astrocytes many neurons survived for a longer period of time and formed long neurites. This support can be explained by the physical properties of astrocytes, which give better attachment to the neurons, and trophic factors secreted by astrocytes that allow for the growth and survival of neurons. As observed earlier, cytokines secreted during insult to the CNS can alter the physical and chemical properties of astrocytes, therefore when neurons are grown on top of TNF- $\alpha$ pre-treated astrocytes, the astrocytes can not give support to the neurons. Few neurons survived for a short period of time and formed shorter neurites.

This finding is in agreement with previous studies by $[29,30]$ which showed that TNF- $\alpha$ activated the apoptotic pathway in neuron (neuroblastma cell line) and human cortical neurons. Using anti-apoptotic drugs inhibited the effect of TNF- $\alpha$. In addition, previous studies by $[31,32]$ showed that TNF- $\alpha$ induced death of motor neurons and inhibited neurites outgrowth and branching of hippocampal neurons. Recent studies showed also that TNF- $\alpha$ contributed to the development of retinal neurodegenerative disorder [33] and neuronal loss in bipolar disorder [34].

Although it is generally accepted that TNF- $\alpha$ has a pro-inflammatory neurotoxic role in neurodegeneration, not all observations confirm this. There are also data suggesting that TNF- $\alpha$ has neuroprotective properties in some pathological conditions such as ischemic brain $[21,35,36]$. More recently it has been reported that TNF$\alpha$ produced by microglia protects the neurons against glutmate toxicity $[37,38]$.

There is evidence that TNF- $\alpha$ has both neurotoxic $[39,40]$ and neuroprotective [41-43] role after ischemic stroke in rats and in mice. It has been suggested that TNF- $\alpha$ may be involved both in the auto propagation of inflammatory processes and cell death and possibly in the more delayed re-constitutive processes of recovery in human of ischemic stroke. In addition, TNF- $\alpha$ has dual effects (Neuroprotective and neurodegeneration) in Par- kinson's diseases [44] and has differential effects on different population of cerebellar neurons as they develop [45].

The finding that TNF- $\alpha$ effects neurite elongation suggests that TNF- $\alpha$ might contribute to inhibiting outgrowth of CNS axons during inflammation or lesion of CNS. This finding is of particular importance because TNF secreted by reactive astrocytes during injury or inflammation of CNS acts as an anti-regenerative factor and prevents axon regeneration after the injury. In addition, TNF- $\alpha$ stimulates the expression of GFAP by astrocytes. Previous studies have suggested that cytokines such as IL-1 and TNF- $\alpha$ act as an astroglia growth factor during brain development and it may play an important role in astrogliosis [46-48]. Following exposure to cytokines (IL-1 $\beta$, TNF- $\alpha$, and IFN- $\gamma$ ) astroglia undergoes functional changes that involve both gene expression and protein synthesis $[18,49]$.

Indeed, in clinically important diseases that lead to elevated production of TNF- $\alpha$ in the CNS and inflammation such as multiple sclerosis and AIDS dementia the therapeutic administration of TNF- $\alpha$ inhibitors may prove to be beneficial [50]. Selective targeting of TNF receptor 1-mediated signalling while sparing TNF-receptor 2 activation may lessen adverse effects of anti-TNF therapies in CNS [51]. It has been reported that a balance between anti-inflammatory neuroprotective factor (neurotrophins) and pro-inflammatory neurotoxic cytokines (TNF) can be used in future therapeutic implications for optic nerve injury, multiple sclerosis and neurodegenerative disorders [52-54]. In conclusion, Astrocytes support the survival of neurons and growth of neurites whereas, TNF- $\alpha$ modulates the astrocytic support of neuronal survival and neurites outgrowth. The molecular mechanism by which TNF- $\alpha$ modulates the astrocytic function needs further investigations.

\section{ACKNOWLEDGEMENTS}

This work is supported by Kuwait University. The author would like to thank Dr. Mai El Mallah, Mrs. Jeena Prashanth and Mr. Ibrahim AbdEl-Barr for technical assistance.

\section{REFERENCES}

[1] Gorden, G.R., Mulligan, S.J. and MacVicar, B.A. (2007) Astrocyte control of the cerebrovasculature. Glia, 55, 1214-1221. doi:10.1002/glia.20543

[2] Prebil, M., Vardjan, N., Jensen, R., Zorec, R. and Kreft, M. (2011) Dynamic monitoring of cytosolic glucose in single astrocytes. Glia, 59, 903-913. doi:10.1002/glia.21161

[3] Furukawa, S., Furukawa, Y. and Satoyoshi, E. (1986) Synthesis and secretion of nerve growth factor by mouse astroglial cells in culture. Biochemical and Biophysical 
Research Communications, 136, 57-63. doi:10.1016/0006-291X(86)90876-4

[4] Yoshida, K. and Gage, F.H. (1992) Cooperative regulation of nerve growth factor synthesis and secretion in fibroblasts and astrocytes by fibroblast growth factor and other cytokines. Brain Research, 569, 14-25. doi:10.1016/0006-8993(92)90364-F

[5] Marchetti, B. (1997) Cross-talk signals in the CNS: Role of neurotrophic and Hormonal factors, adhesion molecules and intercellular signalling agents in leuteinizing hormone (LHRH)-astroglial interactive network. Frontiers in Bioscience, 1, 88-125.

[6] Friedman, W.J., Larkfors, L., Ayer-leliever, C., et al. (1990) Regulation of B-nerve growth factor expression by inflammatory mediators in hippocampal cultures. Journal of Neuroscience Research, 27, 374-382. doi:10.1002/jnr.490270316

[7] Liddell, J.R., Robinson, S.R., Dringen, R. and Bishop, G.M. (2010) Astrocytes retain their antioxidant capacity into advanced old age. Glia, 58, 1500-1509.

[8] McGraw, J., Hiebert, G.W. and Steeves, J.D. (2001) Modulating astrogliosis after neurotrauma. Journal of Neuroscience Research, 63, 109-115. doi:10.1002/1097-4547(20010115)63:2<109::AID-JNR1 $\underline{002>3.0 . \mathrm{CO} ; 2-\mathrm{J}}$

[9] Morrison, R.S., DeVellis, J., Bradshaw, R.A. and Eng, L.F. (1985) Hormones and growth factors induce the synthesis of glial fibrillary acidic protein in the rat brain astrocytes. Journal of Neuroscience Research, 14, 167176. doi:10.1002/jnr.490140202

[10] Laping, N.J., Teter, B., Nichols, N.R., Rozovsky, I. and Finch, C.E. (1994) Glial fibrillary acidic protein: Regulation by hormones, cytokines, and growth factors. Brain Pathology, 1, 259-275. doi:10.1111/j.1750-3639.1994.tb00841.x

[11] Wanner, I.B., Deik, A., Torres, M., et al. (2008) A new in vitro model of glial scar inhibits axon growth. Glia, 56, 1691-1709. doi:10.1002/glia.20721

[12] Sofroniew, M.V. (2005) Reactive astrocytes in neural repair and protection. Neuroscientist, 11, 400-707. doi:10.1177/1073858405278321

[13] Rolls, A., Shechter, R. and Schwartz, M. (2009) The bright side of the glial scar in CNS repair. Nature Reviews Neuroscience, 10, 235-241. doi:10.1038/nrn2591

[14] Lu, P., Jones, L.L. and Tuszynshi, M.H. (2007) Axon regeneration through scars and into sites of chronic spinal cord injury. Experimental Neurology, 203, 8-21. doi:10.1016/j.expneurol.2006.07.030

[15] Faulkner, J.R., Hermann, J.E., Woo, M.J., et al. (2004) Reactive astrocytes protect tissue and preserve function after spinal cord injury. The Journal of Neuroscience, 24, 2143-2155. doi:10.1523/JNEUROSCI.3547-03.2004

[16] John, G.R., Lee, S.C., Song, X., Rivieccio, M. and Brosnan, C.F. (2005) IL-1-regulated responses in astrocytes: Relevance to injury and recovery. Glia, 49, 161-176. doi:10.1002/glia.20109

[17] Hopkins, S.J. and Rothwell, N.J. (1995) Cytokines and nervous system. I: Expression and recognition. Trends in
Neurosciences, 18, 83-85. doi:10.1016/0166-2236(95)93881-W

[18] Benveniste, E.N. (1992) Inflammatory cytokines within the central nervous system: Sources, function and mechanism of action. American Journal of Physiology, 263, c1-c16.

[19] Neumann, H. (2001) Control of glial immune function by neurons. Glia, 36, 191-199. doi:10.1002/glia.1108

[20] Skaper, S.D. (2007) The brain as a target for inflammatory processes and neuroprotective strategies. Annals of the New York Academy of Sciences, 1122, 23-34. doi:10.1196/annals.1403.002

[21] Saha, R.N. and Pahan, K. (2003) Tumor necrosis factor-alpha at the crossroads of neuronal life and death during HIV-associated dementia. Journal of Neurochemistry, 86, 1057-1071. doi:10.1046/j.1471-4159.2003.01942.x

[22] Little, A.R. and O'Callagha, J.P. (2001) Astrogliosis in adult and developing CNS: Is there a role for poinflammatory cytokines? Neurotoxicology, 22, 607-618. doi:10.1016/S0161-813X(01)00032-8

[23] Park, K.M. and Bowers, W.J. (2010) Tumor necrosis factor-alpha mediated signaling in neuronal homeostasis and dysfunction. Cell Signal, 22, 977-983. doi:10.1016/j.cellsig.2010.01.010

[24] Abd-El-Basset, E.M., Kalnins, V.I., Subrahamanyan, L., Ahmed, I. and Fedoroff, S. (1988) 48 Kilodalton intermediate filament-associated protein in astrocytes. Journal of Neuroscience Research, 19, 1-13. doi:10.1002/jnr.490190102

[25] Zhang, S. and Fedoroff, S. (1996) Neuron-microglia interactions in vitro. Acta Neuropathologica, 91, 385-395. doi:10.1007/s004010050440

[26] Bradford, M.M. (1976) A rapid and sensitive method for quantification of microgram quantities of protein utilizing the principles of protein-dyebinding. Analytical Biochemistry, 72, 248-254. doi:10.1016/0003-2697(76)90527-3

[27] Laemmli, U.K. (1970) Cleavage of structural proteins during assembly of head of the bacteriophage T4. Nature, 227, 680-685. doi:10.1038/227680a 0

[28] Towbin, H., Staehlin, T. and Gordon, J. (1979) Electrophoretic transfer of proteins from polyacrylamide gels to nitrocellulose sheets: Procedure and some applications. Proceedings of the National Academy of Sciences, 76, 4350-4354. doi:10.1073/pnas.76.9.4350

[29] Medina, S., Martinez, M. and Hernanz., A. (2002) Antioxidants inhibit the human cortical neuron apoptosis induced by hydrogen peroxide, tumor necrosis factor alpha, dopamine and beta-amyloid peptide 1-42. Free Radical Research, 36, 1179-1184. doi:10.1080/107157602100006445

[30] Miliani, D., Zauli, G., Rimondi, E., et al. (2003) Tumour necrosis factor-related apoptosis-inducing ligand sequentially activates pro-survival and pro-apoptotic pathway in SK-N-MC neuronal cells. Journal of Neurochemistry, 86, 126-135. doi:10.1046/j.1471-4159.2003.01805.x

[31] Neumann, H., Schweigreiter, R., Yamashita. T. et al., 
(2002) Tumour necrosis factor inhibits neurite outgrowth and branching of hippocampal neurons by a Rh-dependent mechanism. The Journal of Neuroscience, 22, 854862.

[32] Li, B., Xu, W., Luo, C., Gozal, D. and Liu, R. (2003) VEGF-induced activation of the $\mathrm{P} 23-\mathrm{K} / \mathrm{Akt}$ pathway reduces mutant SOD1-mediated motor neuron cell death. Molecular Brain Research, 17, 155-164. doi:10.1016/S0169-328X(03)00025-1

[33] Al-Gayyar, M.M. and Elsherbiny, N.M. (2013) Contribution of TNF- $\alpha$ to the development of retinal neurodegenerative disorders. European Cytokine Network, 24, 27-36.

[34] Brietzke, E. and Kapczinski, F. (2008) TNF-alpha as a molecular target in bipolar disorder. Progress in NeuroPsychopharmacology \& Biological Psychiatry, 32, 13551361. doi:10.1016/j.pnpbp.2008.01.006

[35] Brabers, N.A. and Nottet, H.S. (2006) Role of the proinflammatory cytokines TNF-alpha and IL-1beta in HIVassociated dementia. European Journal of Clinical Investigation, 36, 447-458. doi:10.1111/j.1365-2362.2006.01657.x

[36] Lambertsen, K.L., Clausen, B.H., Babcock, A.A., et al. (2009) Microglia protect neurons against ischemia by synthesis of tumor necrosis factor. The Journal of Neuroscience, 29, 1319-1330. doi:10.1523/JNEUROSCI.5505-08.2009

[37] Suzuki, T., Hide, I., Kohsaka, S., Inoue, K. and Nakata, Y. (2004) Production and release of neuroprotective tumor necrosis factor by $\mathrm{P} 2 \mathrm{X}_{7}$ receptor-activated microglia. The Journal of Neuroscience, 7, 1-7. doi:10.1523/JNEUROSCI.3792-03.2004

[38] Dolga, A.M., Grank, T., Knaus, H.G., et al. (2008) TNFalpha-mediates neuroprotection against glutamate-induced excitotoxicity via NF-KappaB-dependent up-regulation of K2.2 channels. Journal of Neurochemistry, 107, 1158-1167.

[39] Barone, F.C., Arvin, B., White, R.F., Miller, A., Webb, C.L., Willette, R.N., Lysko, P.G. and Feuerstein, G.Z. (1997) Tumor necrosis factor-alpha. A mediator of focal ischemic brain injury. Stroke, 28, 1233-1244. doi:10.1161/01.STR.28.6.1233

[40] Dawson, D.A., Martin, D. and Hallenbeck, J.M. (1996) Inhibition of tumor necrosis factor-alpha reduces focal cerebral ischemic injury in the spontaneously hypertensive rat. Neuroscience Letters, 218, 41-44. doi:10.1016/0304-3940(96)13116-5

[41] Nawashiro, H., Martin. D. and Hallenbeck, J.M. (1997) Neuroprotective effects of TNF binding protein in focal cerebral ischemia. Brain Research, 778, 265-271. doi:10.1016/S0006-8993(97)00981-5

[42] Sairanen, T., Lindsberg, M., Paetau, A., Kaste, M. and Lindsberg, P. (2001) Evolution of cerebral tumor necrosis factor alpha production during human ischemic stroke. Stroke, 32, 1750-1773. doi:10.1161/01.STR.32.8.1750

[43] Bernardino, L., Agasse, F., Silva, B., Ferreira, R., Grade,
S. and Malva, J.O. (2008) Tumor necrosis factor-alpha modulates survival, proliferation, and neuronal differentiation in neonatal subventricular zone cell cultures. Stem Cells, 26, 2361-2371. doi:10.1634/stemcells.2007-0914

[44] Chertoff, M., Di Paolo, N., Schoeneberg, A., et al. (2011) Neuroprotective effects of chronic expression of tumor necrosis factor $\alpha$ in the nigrostriatal dopaminergic circuit of adult mice. Experimental Neurology, 227, 237-251. doi:10.1016/j.expneurol.2010.11.010

[45] Oldreive, C.E. and Doherty, G.H. (2010) Effect of tumour necrosis factor-alpha on developing cerebellar granule and purkinje neurons in vitro. Journal of Molecular Neuroscience, 42, 44-52. doi:10.1007/s12031-010-9370-9

[46] Lachman, L.B., Brown, D.C. and Dinarello, C.A. (1987) Growth promoting effect of recombinant interleukin-1 and tumor necrosis factor for a human astrocytoma cell line. Journal of Immunology, 138, 2913-2916.

[47] Giulian, D., Woodward, J., Young, D.G., Krebs, J.F. and Lachman, L.B. (1988) Interleukin-1 injected into mammalian brain stimulates astrogliosis and neovascularization. Journal of Neuroscience, 8, 2485-2490.

[48] Abd-El-Basset, E.M. and Abd-El-Barr, M.M. (2011) Effect of interleukin- $\beta$ on the expression of actin isoforms in cultured mouse astroglia. Anatomical Record, 294, 16-23. doi:10.1002/ar.21303

[49] Merrill, J.E. (1992) Tumor necrosis factor- $\alpha$, interleukin-1 and related cytokines in brain development: Normal and pathological. Developmental Neuroscience, 14, 1-10. doi: $10.1159 / 000111642$

[50] Venters, H., Tang, Q., Liu, Q., et al. (1999) A new mechanism of neurodegeneration: A proinflammatory cytokine inhibits receptor signaling by a survival peptide. Neurobiology, 96, 9879-9884.

[51] McCoy, M.K. and Tansey, M.G. (2008) TNF signaling inhibition in the CNS: Implications for normal brain function and neurodegenerative disease. Journal of $\mathrm{Neu}$ roinflammation, 5, 45-57. doi:10.1186/1742-2094-5-45

[52] Tabakman, R., Lecht, S., Sephanova, S., Arien-Zakay, H. and Lazarovici, P. (2004) Interactions between the cells of the immune and nervous systems: Neurotrophins as neuroprotection mediators in CNS injury. Progress in Brain Research, 146, 387-401. doi:10.1016/S0079-6123(03)46024-X

[53] Marchetti, B. and Abbracchio, M.P. (2005) To be or not to be (inflamed) - Is that the question in anti-inflammatory drug therapy of neurodegenerative disorders? Trends in Pharmacological Science, 26, 517-525. doi:10.1016/j.tips.2005.08.007

[54] Tweedie, D., Sambamurti, K. and Greig, N.H. (2007) TNF-alpha inhibition as a treatment strategy for neurodegenerative disorders: New drug candidates and targets. Current Alzheimer Research, 4, 378-385. doi: $10.2174 / 156720507781788873$ 